\title{
Book Review: Moving to A Posthuman Technosphere
}

\section{Steven Umbrello}

Institute for Ethics and Emerging Technologies

Review of Tecnosfera: Proiezioni per un future postumano, by Roberto Marchesini (Castelvecchi, 2017); 256 pp., paperback, €22.00, ISBN 9788832821680.

Historically, all narrative accounts of human being and identity take the form of a journey, a clear-cut and wholesome navigation around explicitly defined lands and territories. This causal holism - both for the genesis and for the genealogy of geographies - is what the Greeks termed techne, and art, craftsmanship or competence to bring an idea into being. Similarly, then techne is also aletheia, a revelation of something that is obscured or hidden and as such, it is also apate, a deceit. The project group's second major achievement was to introduce a new and fruitful approach to the metaphysics of technical artefacts. This approach grew out of dissatisfaction with previous accounts that were found to be inadequate. On the one hand, technical artefacts have often been described in merely physical and structural terms. That is clearly insufficient. You have not understood what a set of chimes is if you only know how it is constructed (with hanging metal tubes of different lengths). A set of chimes has to be understood in relation to its function as a musical instrument.

This is how the Italian philosopher, ethologist and prolific writer begins Tecnosfera: Proiezioni per un future posthuman (literally Technosphere: Projections for a posthuman future). In doing so, Marchesini aims to explore the question of what the future of human beings is. To do this, he takes an unorthodox approach by exploring the concept of 'technique' (etymologically related to technology and techne) and as such may be able to anticipate better what may be waiting in the future.

The opening chapter begins by reconstructing the ominous homo faber (the building-human/tool-using human) and its techno-poetic disposition. This conception is starkly contrasted to that of the non-human, often background and 
ambient dimension of the animal world: the controllers $\mathrm{v}$. the controlled (or builtupon). Doing this, Marchesini draws upon the more extensive posthumanist discourse on the critique of humanist dichotomies, i.e., man/animal, culture/nature, etc. What he argues, however, is somewhat more nuanced than what a lot of other topical texts tangle with. He argues that the techno-mediated praxis that has enabled humans to discard and use the ecological work is often juxtaposed with the world of animality which is controlled through the application of techne. The former is rarely if ever put under scrutiny. However, the world of animality is often subjected to criticism in when its existence becomes inconvenient to the other.

His aim, then, is to show how this division is erroneous given its misinterpretation of techne that humanism has attributed to it. The humanistic conception of technology as something that adds to a human deficiency that the human is created posterior to the technology is in direct error to the reality that Marchesini proposes. Techne, then, is not human-based; instead, humanity as such is pushed to the periphery and decentred from the humanist pedestal of superiority. This theologically generated gravitation centre for the human subject dissolves, and the contingencies of the entanglements with other forms of life are unveiled. To this end, techne is anthropo-decentrive and an unveiling of animality.

The vision of the future opens up on the hinge of infinite contingencies, and the decentred human no longer has a substantial centre foundation from which it can dominate. Issues arise, however, it is difficult to escape the clutches of humanism and its philosophical impedimenta that the instruments of techne are passive slaves for the workings of humankind, things that can compensate for inherent shortcomings.

Regardless, the genuine ecological crisis, the economic realities and disparities provide an urgency for novel thinking and imagination of possible futures. This transition point that we find ourselves in force us to reflect on techne and our relationship to it. To do this, Marchesini draws on the symbolical figure of the cyborg that is foundational to posthumanist discussions. The cyborg is emblematic because the conception of technology as a tool becomes untenable as a corporal and virtual integration make the boundaries of fleshy existence strange and jagged. This unique existential space of agency permits a plurality of phenomenologies to encounter one another. This is the Technosphere.

In sum, Marchesini's project is not that of critiquing techne per se, but to critique the humanist appropriation of the term and its material consequences. A re-imagination of techne in light of modern scientific advances, particularly in those of cybernetics and virtual reality, reveal and opening-up of the world rather than a closing down and separation of humanity from other forms of life. Enabling this novel way of thinking encourages novel future modalities to emerge. 
Marchesini's project is unquestionably grandiose, and for the most part, he is successful in providing convincing historical, technical and genealogically arguments for his thesis. However, the text does get bogged down in what can be characterized as an increasingly complex and systematic set of technical references. To that end, Tecnosfera is not aimed at a general audience but preferably those who are already familiar with much of the existent posthumanist discourse. This is exacerbated by the fact that the text currently exists only in its original Italian, which is a shame given the immensity and novelty of thought presented by Marchesini.

Other oddities present themselves also, such as the positioning of the table of contents at the back of the book under the title of 'index' and the curious lack of foundational texts. Regarding the latter, only passing mention is made of the work by Donna Haraway, and even then it is only her Cyborg Manifesto (1995). To be fair the manifesto is of particular relevance to Marchesini's aim, but her later works develop her original thesis to a greater extent as do the countless other works that constitute the 'cyborg discourse' that ensued from the manifesto's publication. Similarly, no work from Bruno Latour or Timothy Morton is cited or mentioned. The failure to evoke the former's work is particularly strange given Latour's flat ontology which is often discussed as the primary philosophical ontology for the decentering of the Anthropos.

Regardless, Technosfera's unique approach to posthumanism is a fresh and welcome addition to the ongoing discussions in philosophy, critical theory, anthropology and literary criticism among others. Although Marchesini provides many hypotheses regarding possible futures, he is clear that this reworking of techne makes any certainty futile given the openness of the future. To this end, the revaluation of techne in Tecnosfera and its philosophical implications is where the real interest in the text lies, and it is not disappointing. Because of that, the book is definitely worth reading if one has the language capacity, but even then it will be a challenge given Marchesini's technical and stylistic depth. If the book were available in other languages for dissemination, there would be little that can impede its endorsement. 
